\title{
Experimental setup for rapid crystallization using favoured chemical potential and hydrodynamic conditions
}

\author{
V K DIXIT, B V RODRIGUES and H L BHAT* \\ Department of Physics, Indian Institute of Science, Bangalore 560 012, India
}

MS received 9 April 2001; revised 2 July 2001

\begin{abstract}
The rapid crystallization of $\mathrm{KH}_{2} \mathrm{PO}_{4}$ (KDP) from solution is demonstrated at a rate up to $\approx 7.5 \mathrm{~mm} /$ day along [100] and $22 \mathrm{~mm} /$ day along [001] in a crystallizer of $5 \mathrm{l}$ capacity, using accelerated crucible rotation technique (ACRT) and simulated platform geometry for controlling the hydrodynamic conditions. On an experimental basis we have grown the crystals up to $40 \times 43 \times 66 \mathrm{~mm}^{3}$ size in about 3 days. Comparative analysis of the main structural and optical properties of crystals grown by conventional and rapid crystallization technique, is discussed.
\end{abstract}

Keywords. Rapid crystallization; ACRT; hydrodynamics.

\section{Introduction}

The crystal growth from solution is widely used because it presents several advantages. For example, it is often easier to setup such a facility than other methods like melt growth, flux growth and growth from vapour. Also, since it is a low temperature technique it results in high quality single crystals. However, the growth rate achieved in conventional solution growth is relatively low, i.e. $\approx 1-$ $2 \mathrm{~mm} /$ day while in melt growth technique it can be as high as $\approx 20 \mathrm{~mm} / \mathrm{h}$. In order to increase the growth rate in the solution growth technique several efforts have been made, mainly on KDP because of its utility in large sized frequency conversion cells to be used for the inertial confinement fusion experiments (Rhodes et al 1993; Lindl 1995). In fact, at present, $\mathrm{KH}_{2} \mathrm{PO}_{4}$ (KDP) is the only inorganic nonlinear material which is grown to very large (50-60 cm) lateral dimensions (Zaitseva et al 1997). Growth of such large crystals takes about one year by the conventional method, resulting in higher costs due to the long-term labour and machine running. The rapid crystal growth to decrease the cost and growth period needs higher level of supersaturation and suppression of spontaneous crystallization in the mother solution. However, at such high supersaturation levels solution stability depends on many parameters such as overheating of the solution, purity of raw materials, crystallizer design, hydrodynamic conditions, rate of temperature reduction, etc. In the present paper we describe a small scale experimental setup for rapid crystallization in which growth is favoured by chemical potential and hydrodynamic conditions. Using

\footnotetext{
*Author for correspondence
}

this we have grown $\mathrm{KH}_{2} \mathrm{PO}_{4}$ (KDP) single crystals up to $40 \times 43 \times 66 \mathrm{~mm}^{3}$ in dimensions on a point seed in a glass crystallizer of 51 capacity in about $72 \mathrm{~h}$. We also present the comparative study of structural and optical properties of traditionally and rapidly grown KDP crystals.

\section{Experimental}

The experimental arrangement for growing crystals by rapid crystallization process is schematically shown in figure 1. Referring to the figure, the solution is contained in a 51 cylindrical flask $F$ with a lid $\mathrm{L}$ to which teflon platform $\mathrm{P}$ is anchored through a ball bearing $\mathrm{B}$. The internal solution is stirred by this teflon platform at a speed of $150 \mathrm{rpm}$ to $300 \mathrm{rpm}$. For the favoured global mixing of the solution in radial and axial directions during growth, the design of the platform is modified, specially with regard to the vertical post of the platform as shown in figure 2. The beneficial effect of this geometry will be discussed in the crystal growth section. Stirring of the solution achieved by the accelerated seed rotation technique (ACRT), also helps to increase the growth rate in lateral directions as well as change in morphology of the crystal because of forced solution convection. The typical ACRT cycle and the relevant circuit are shown in figure 3. The main feature of this circuit is the use of XR-8038, which is a precision waveform generator IC capable of producing sine, square, triangular, saw-tooth and pulse waveforms with variable operating frequency. We have used here triangular waveform as input in amplifying stage built using a complementary pair of power transistors (3005 type), the output of which drives the d.c. motor $\mathbf{M}_{3}$. The solution of the cylindrical flask is heated by the 
external 201 water bath W. Large volume of the bath which is continuously stirred by the stirrer connected to motor $M_{1}$ prevents rapid temperature fluctuations and hence ensures very good temperature control. The thermostatic bath is heated with an infrared lamp I at the base of the unit and the control is affected by a long range electrical adjustable contact thermometer T. The supersaturation is achieved by driving the contact of the electrical thermometer to lower temperature using a synchronous motor $\mathrm{M}_{2}$ attached to the magnetic setting device. The rate at which the temperature is lowered can be varied by means of a cam operated time sharing device which switches the contact thermometer drive motor on for varying period of time depending on the disposition of the cam relative to the microswitch. The associated

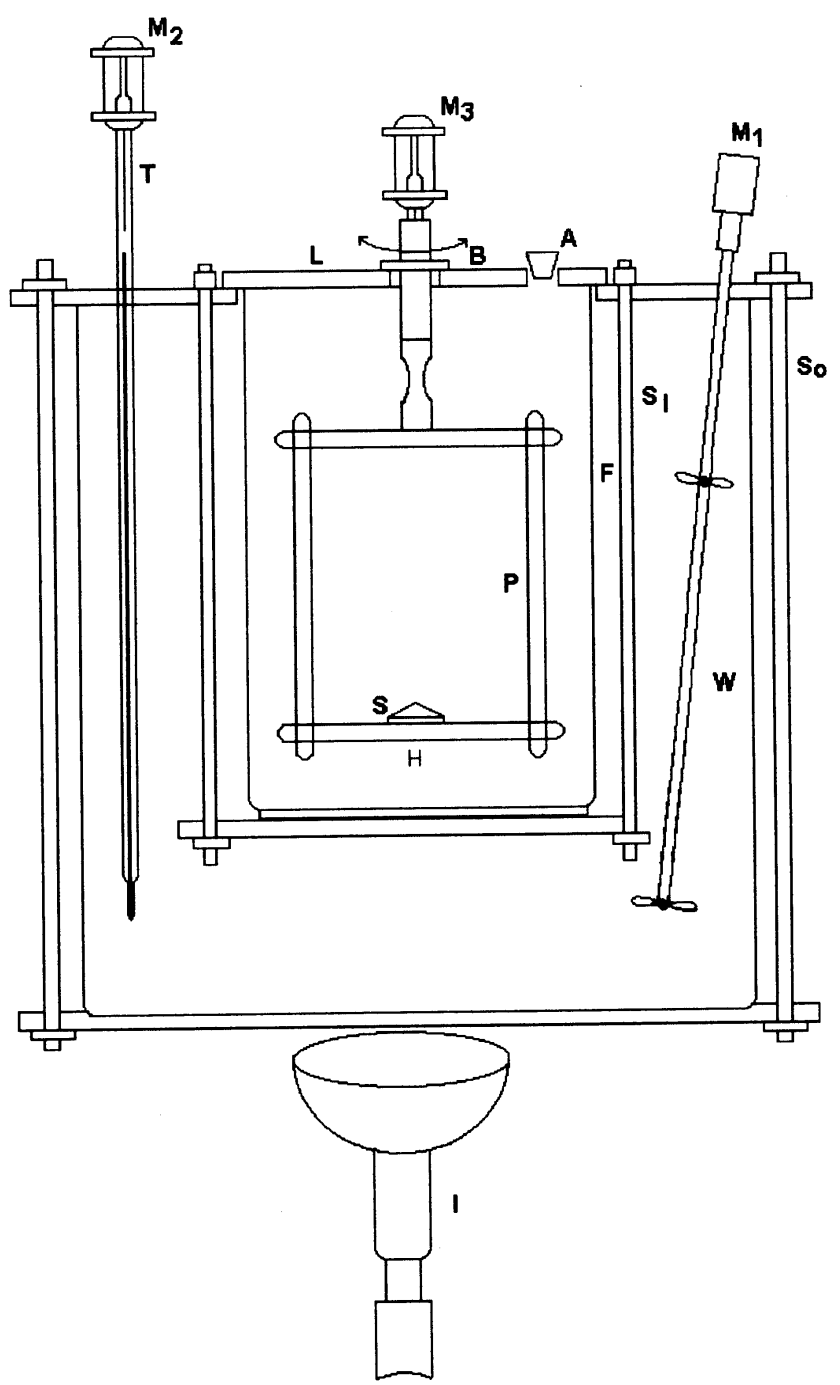

Figure 1. Schematic of the crystallizer unit used for rapid crystallization (I, IR lamp; $S_{0}$, outer support stand; $S_{i}$, inner support stand; S, seed; $\mathrm{M}_{1}$, stirrer motor; W, water bath; $\mathrm{F}$, cylindrical flask; P, teflon platform; A, air sealed lid; B, ball bearing; L, lid; $\mathrm{T}$, contact thermometer; $\mathrm{M}_{2}$, synchronous motor; $\mathrm{M}_{3}, 12 \mathrm{~V}$ d.c. motor). electronic circuit has been built for the purpose of controlling the temperature.

\section{Crystal growth}

On an experimental basis the growth of $\mathrm{KH}_{2} \mathrm{PO}_{4}$ (KDP) single crystal was carried out. The solution was prepared using triple distilled water obtained by distilling deionized water. AR grade $\mathrm{KH}_{2} \mathrm{PO}_{4}(\mathrm{KDP})$ material was purified by repeated crystallization before preparing the mother solution. This process was followed principally to remove the metal ion impurities such as $\mathrm{Al}, \mathrm{Fe}, \mathrm{Cr}$ which can easily get incorporated into the prismatic sectors and deteriorate the optical quality of the crystals. For removing suspended impurities, the solution was subjected to centrifuge technique at $15000 \mathrm{rpm}$. The resulting solution was filtered with a micropore filter (pore size $\approx 0.2 \mu \mathrm{m}$ ) and transferred to crystallizer for growth.

\subsection{Influence of overheating on growth}

The rate of crystal growth is determined by several steps in the growth process. This can either be the diffusion of materials to the surface or the kinetics of surface processes. Both the diffusion and surface integration kinetics are processes driven by difference in chemical potential. Hence the driving force for the crystal growth is given as

$$
\mu_{\mathrm{s}}-\mu_{\mathrm{s}}^{*}=v R T \ln \left(a_{\mathrm{s}} / a_{\mathrm{s}}^{*}\right),
$$

where $a_{\mathrm{s}}, a_{\mathrm{s}}^{*}$ are the activities of the supersaturated and saturated ions, $\mu_{\mathrm{s}}, \mu_{\mathrm{s}}^{*}$ are the respective chemical potentials and $v$ the stoichiometric coefficient of the ions. For diffusion-limited processes the diffusion coefficient is an
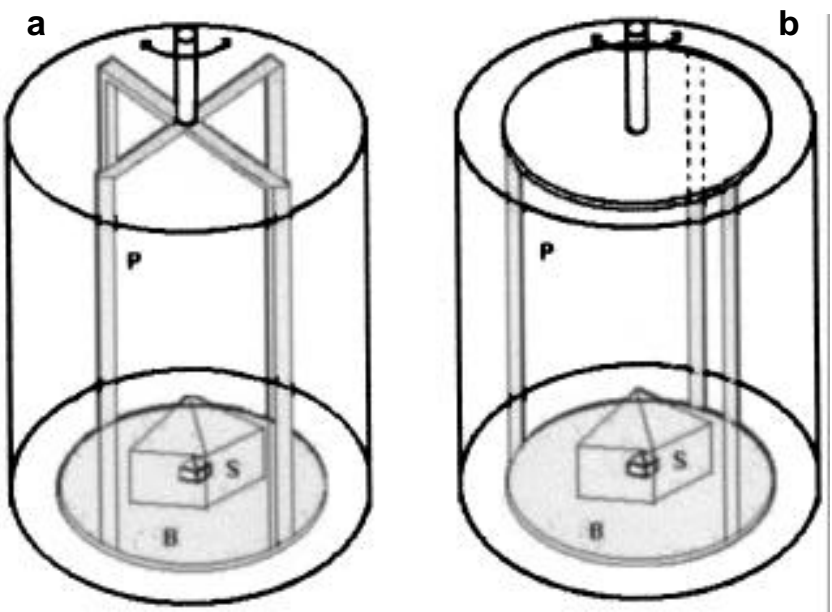

Figure 2. Schematic diagrams of teflon platform inside the 51 flask with different vertical posts: a. four-post configuration and b. three-post with disc support configuration (B, base; S, KDP seed; C, KDP crystal; P, vertical post). 
important parameter and with earlier study it is known that it is a decreasing function with time. The variation of the diffusion coefficient with time is believed to be due to the formation of subcritical solute crystals. Their average size grows larger as the solution remains in the metastable state. Evidence for such clusters was first reported by Mullin and Leci (1969). The average cluster size can range between 2 and 100 solute molecules though most of the clusters were dimers or trimers (Myrson and Lo 1990). In KDP, Cerreta and Berglund (1987) used Raman spectroscopy and found Raman peaks which suggest that the dihydrogen phosphate anions are forming polymers through chains of hydrogen bonds and these polymers with associated positive ions may be the growth units in crystal growth. These clusters can be removed by overheating the solution or by giving additional energy to the solution by an acoustic power (Nakatsuka et al 1997) because at normal supersaturation level only a fraction of molecules are dissolved completely and some of the undissolved molecules will remain in the solution as clusters which can not be visibly seen. Energy supplied to the solution by these procedures can dissociate the clusters. Hence for removing the invisible micro-clusters, one can give an excess thermal energy

$$
E=\frac{3}{2} n k \Delta T
$$

by overheating, where $n$ is the number of molecules in a cluster, $k$ the Boltzmann constant and $\Delta T$ the difference between overheating temperature and saturation temperature. The overheating conditions typically applied in the present rapid crystallization are as follows: Firstly the solution is overheated to $35^{\circ} \mathrm{C}$ above the saturated temperature, $T_{\mathrm{s}}=45^{\circ} \mathrm{C}$ and kept for a time, $t=72 \mathrm{~h}$. After this overheating duration the solution temperature was brought back to temperature, $T_{\mathrm{s}}$, and the growth was initiated. We have also observed that when the solution was not subjected to overheating, the probability of the formation of spontaneous nucleation was much more compared to the overheated solution. As a result, crystals grow at various places inside the crystallizer. Consequently, the availability of the ejected material near the seed crystal is less, leading to negligible growth rate along [100] and [010] direction as compared to [001] direction.

\subsection{Influence of improved platform design on growth}

The kinetics of the surface processes is strongly dependent on convection driven by crystal and platform

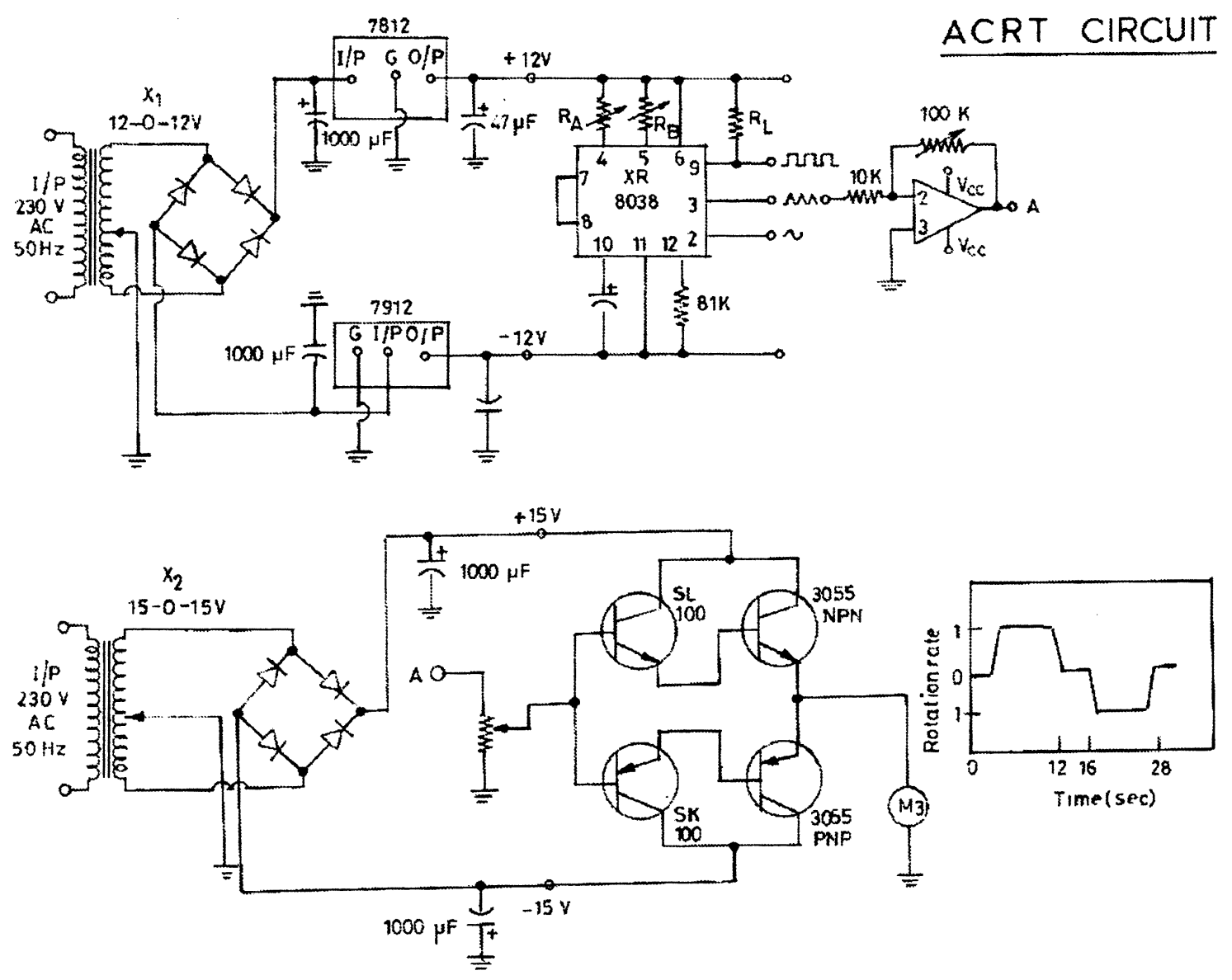

Figure 3. The circuit for accelerated seed rotation and rotation cycle. 
Table 1. Summary of typical growth experiments.

\begin{tabular}{|c|c|c|c|c|c|c|c|}
\hline Run no. & $\begin{array}{l}\text { Platform } \\
\text { geometry }\end{array}$ & $\begin{array}{l}\text { Temperature } \\
\text { range of growth } \\
\left({ }^{\circ} \mathrm{C}\right)\end{array}$ & $\begin{array}{l}\text { Cooling rate } \\
\left({ }^{\circ} \mathrm{C} / \text { day }\right)\end{array}$ & $\begin{array}{l}\text { Rotation rate } \\
\text { of the platform } \\
(\mathrm{rpm})\end{array}$ & $\begin{array}{l}\text { Growth rate } \\
\text { along } \\
(\mathrm{mm} / \text { day })\end{array}$ & $\begin{array}{l}\text { Size of the } \\
\text { grown crystals } \\
\left(\mathrm{mm}^{3}\right)\end{array}$ & Remarks \\
\hline \# 43 & $\begin{array}{l}\text { Three posts } \\
\text { with disc } \\
\text { support }\end{array}$ & $45-36$ & 3 & 150 & $\begin{aligned} a & =3 \cdot 0 \\
b & =2 \cdot 5 \\
c & =25\end{aligned}$ & $29 \times 27 \times 75$ & $\begin{array}{l}\text { Asymmetric with respect } \\
\text { to point seed }\end{array}$ \\
\hline \# 50 & Four posts & $45-36$ & 3 & 150 & $\begin{aligned} a & =7 \cdot 5 \\
b & =7 \cdot 0 \\
c & =22\end{aligned}$ & $43 \times 40 \times 66$ & $\begin{array}{l}\text { Symmetric with respect } \\
\text { to point seed }\end{array}$ \\
\hline
\end{tabular}
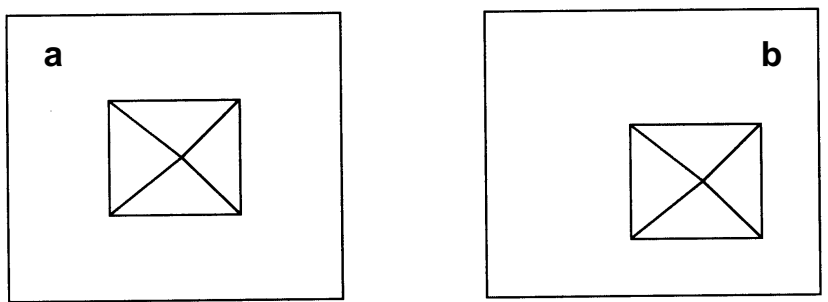

Figure 4. Schematic diagram of the cross section of grown crystals with different vertical post geometry: a. four-post support geometry and b. three-post with disc support geometry.

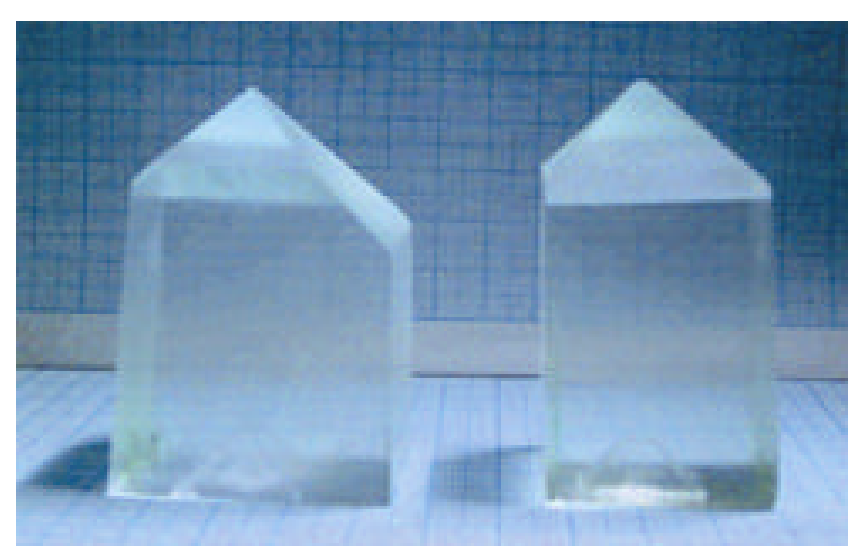

Figure 5. KDP crystals grown in three days.

rotation. Such stirring strategies have been used before to enhance growth rates in many solution growth systems (Wilcox 1983). However, the speed form of the flow field through the system is important for the control of deleterious effects, such as unwanted modification of the crystal growth habit, spurious nucleation events and morphological instabilities of the growing crystal. Here we used two different vertical post support configurations as shown in figure 2. We observed that the cross section of the grown crystal was asymmetric in the three-post with disc support while in four-post support it was more symmetric with respect to the seed crystal (see schematic diagram in figure 4). This may be because the four-post support gives symmetric flow pattern in $\mathrm{X}-\mathrm{Y}$ plane, and the pressure exerted on crystal interface with this design will be uniform in all directions which is also corroborated by other

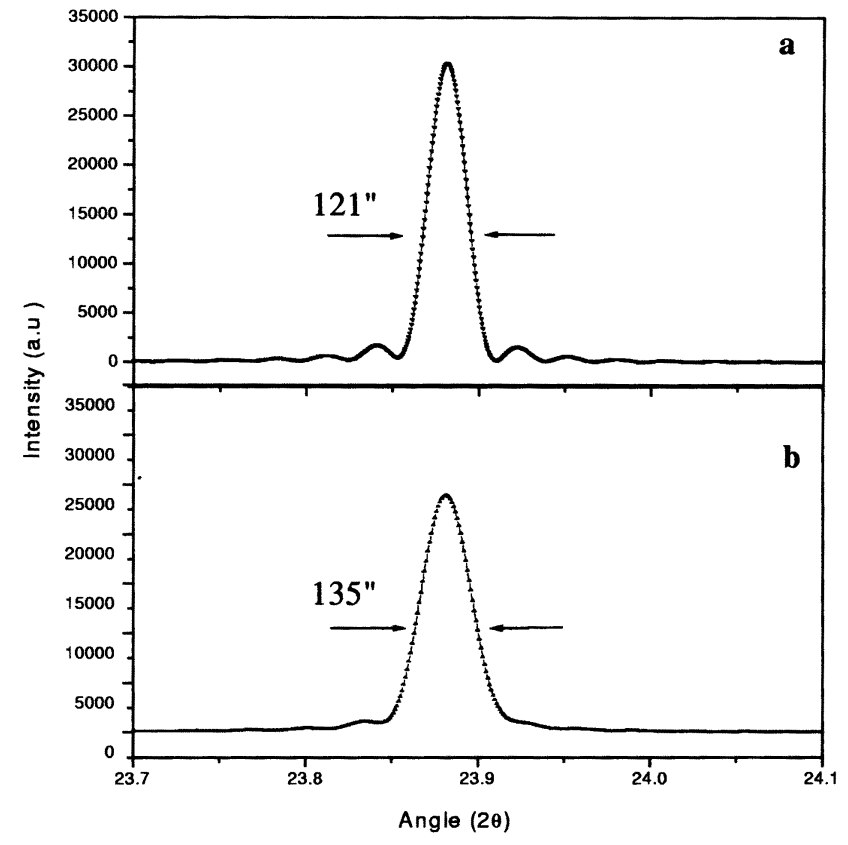

Figure 6. X-ray rocking curves for (100) oriented plate of a. conventionally grown and $\mathbf{b}$. rapidly grown KDP crystal.

simulation studies (Zhou and Derby 1997; Yeckel et al 1998). From these results we conclude that the four-post geometry is more favourable for rapid crystallization. The supersaturation $\sigma$ of the solution at the time of seeding in our growth experiments was calculated by using the formula

$$
\sigma=\left(\left(C_{\mathrm{i}}-C_{\mathrm{f}}\right) / C_{\mathrm{f}}\right) \times 100
$$

where $C_{\mathrm{i}}$ is the initial concentration of the solution at the saturated temperature, $T_{\mathrm{s}}$ and $C_{\mathrm{f}}$, final concentration at temperature, $T_{\mathrm{f}}$ generating spontaneous nucleation and was typically $22 \cdot 1 \%$. The solution temperature was then decreased at a rate of $3{ }^{\circ} \mathrm{C} /$ day. The crystal grew at a rate of $\approx 22 \mathrm{~mm} /$ day along $c$-axis and $\approx 7.5 \mathrm{~mm} /$ day along $a$-axis. The summary and typical growth experiments are given in table 1 and the grown crystals are shown in figure 5 . 


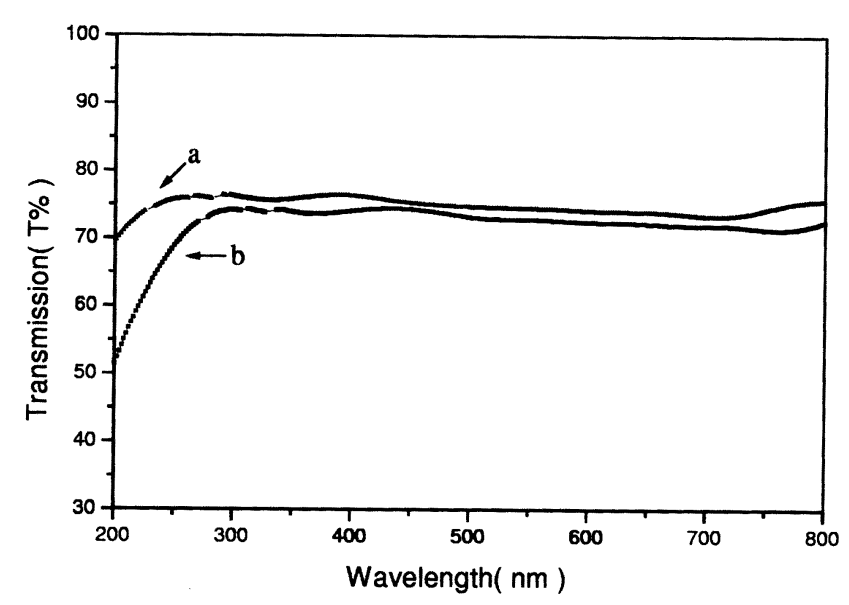

Figure 7. Optical transmission spectra of KDP crystal: a. conventionally grown and $\mathbf{b}$. rapidly grown.

\section{Structural and optical properties}

The X-ray rocking curve measurements were carried out for (200) reflection on both rapidly grown and traditionally grown crystals using a high resolution Siemens D5005 powder diffractometer with $\mathrm{CuK}_{\alpha}$ radiation $(\lambda=1.5418 \AA$ ). The full width at half maximum (FWHM) for the two cases were 121 arc-sec (conventionally grown crystal) and 135 arc-sec (rapidly grown crystal) as can be seen in figure 6. Hence the change in FWHM is only nominal indicating that the crystal quality is comparable.

The optical properties of rapidly grown crystal was compared with that of traditionally grown crystals. The sample of the rapidly grown crystal in the form of $\mathbf{c}$ plate was cut from the region just above the seed cap and the reference sample from traditionally grown crystal was also a c plate. The UV-VIS transmission spectra were recorded for both these samples using Shimadzu spectrophotometer and are shown in figure 7. The absorption coefficient for rapidly grown crystal was found to be $0.64 \mathrm{~cm}^{-1}$ at $248 \mathrm{~nm}$ and that for traditionally grown crystal was $0.47 \mathrm{~cm}^{-1}$ at $248 \mathrm{~nm}$. The absorption coefficient is slightly higher for the rapidly grown crystals which could be due to the incorporation of ppm level metal ion impurities in the lattice which may also be responsible for the apparent shift in the absorption edge
(Zaitseva et al 1991; Amandosov et al 1998) seen in figure 7 .

\section{Conclusions}

We have fabricated a 51 capacity solution growth setup for rapid crystallization with favoured chemical potential and hydrodynamic conditions. On an experimental basis we have grown $\mathrm{KH}_{2} \mathrm{PO}_{4}(\mathrm{KDP})$ crystals up to $40 \times 43 \times$ $66 \mathrm{~mm}^{3}$ size in about 3 days. Preliminary X-ray and optical characterization were carried out which revealed that there is no significant difference between the crystals grown by rapid crystallization process and traditional method.

\section{Acknowledgement}

One of the authors (VKD) acknowledges CSIR, New Delhi, for the award of a senior research fellowship (SRF).

\section{References}

Amandosov A T, Pashina Z S and Rashkovich L N 1983 Sov. J. Quantum Electron. 13271

Cerreta M K and Berglund K A 1987 J. Cryst. Growth 84577

Lindl J D 1995 Phys. Plasmas 23933

Mullin J W and Leci C L 1969 Philos. Mag. 191075

Myrson A S and Lo P Y 1990 J. Cryst. Growth 991048

Nakatsuka M, Fujioka K, Kanabe T and Fujita H 1997 J. Cryst. Growth 171531

Rhodes M A, De Yoreo J J, Woods B W and Atherton L J 1993 ICF Annual Report of Lawrence Liwermore National Laboratory, UCRL-LR-105820 9293

Wilcox W L 1983 J. Cryst. Growth 65133

Yeckel A, Zhou Y, Dennis M and Derby J J 1998 J. Cryst. Growth 191206

Zaitseva N P, Ganikhanov F Sh, Kachalov O V, Efimkov V F, Pastukhov S A and Sobolev V B 1991 Sov. Phys. Crystallogr. 36697

Zaitseva N P, De Yoreo J J, Dehaven M R, Vital R L, Montgomery K E, Richardson M and Atherton L J 1997 J. Cryst. Growth 180255

Zhou Y and Derby J J 1997 J. Cryst. Growth 180497 